P. C. H. Martens, S. Tsuruta, and M. A. Weber, eds.

\title{
Does the Magnetodynamic Model for the Formation of AGN Jets Survive New Findings of High-energy Phenomena near the Central Objects?
}

\author{
Y. Uchida \\ Department of Physics, Science University of Tokyo, Shinjuku-ku, \\ Tokyo 162-8601, Japan
}

\begin{abstract}
Here, we argue that, despite all-new findings of phenomena with very large Lorentz factors, the importance of the magnetodynamic process accelerating and collimating AGN jets should not be affected because there exists evidence in the features of the jets and tails at large distances indicating that the wiggles of the jet structure are likely to be due to a magnetic, helical kink instability. These systematic features require too much energy and coherence of the driving process to be produced locally and are most naturally produced by a magnetic effect coming from the powerful central engine. This indicates that the major effect producing the jets, and the lobes with hotspots at the tips of the jets, is likely to be the Poynting flux carried by torsional Alfvén wave trains plus the reaccelerated high-energy particles in them. The very large Lorentz-factor phenomena should not hinder the transfer of these magnetic effects and are likely to be byproducts of the basic magnetodynamic process, and not the reverse.
\end{abstract}

\section{Introduction}

New findings about very large Lorentz-factor phenomena in the central part of AGNs have been obtained, and some of them seem to suggest that the energy of the magnetic field in them may be small compared with the energy of the pair plasma production in that region. Based on such results, some researchers claim that the role of the magnetic field can not be the major effect there, and that the mechanism of jet production may be something else.

We here argue, based on evidence derived from radio astronomical techniques about the outer parts of the AGN + jets + lobes systems (namely, information about the projected magnetic field directions in the jets and tails, and the Faraday Rotation Measure associated with them), that the above claim may not hold, and that the magnetodynamical process remains essential in the jet production, collimation, et cetera, despite all the new evidence for the very large Lorentz-factor phenomena. 


\section{Radio Astronomical Clues Providing the Basis for Our Argument}

Here, we first argue for the necessity for the magnetodynamic processes to originate near the central object, based on the fact that the magnetic field structure in the radio jets and tails of some AGNs has a very systematic, characteristic distribution indicating that a magnetic field is playing an active role there. Since there are many factors which may degrade such clear signatures even if they exist, like inappropriate viewing angles, the effect of the foreground media covering them, et cetera, the fact that there are a number of AGNs that show such systematic, characteristic signatures of magnetic effects may mean that those are intrinsic in the process producing the jets and tails. Thus, the magnetic action should be taken into account in considering the mechanism causing jets and tails. The signatures we are talking about here are the systematic distributions of the Faraday-Rotation-corrected directions of the projected magnetic field, together with the Faraday Rotation Measure (FRM) itself, in jets and tails at large distances from the central engine of the AGNs.

Can such information about remote parts of the system, like the parts of the jets and tails $\mathrm{kpc}$ to hundred kpc away from the central engine, be used in making such a claim against what are claimed from the high-energy phenomena right near the central engine? We believe "yes", and give some arguments for this in the following.

\subsection{Helical Magnetic Field in the Jets}

The distribution of the directions of the projected magnetic field in the jets and lobes from AGNs has been measured and reported since the 1970s. The directions of the projected magnetic field vectors in the jets in a number of cases are roughly aligned with the jet structure but, in many cases, systematically tilted from the direction of the jet axis, and the tilt angle gets greater if there exist knots in the jets. A typical example (NGC6251) is given in Figure 1. The distribution of the FRM of the same object is given in Figure 1b (both Perley, Bridle, \& Willis 1984).

Another well-known example is M87. The projected field directions are again tilted (Owen, Hardee, \& Cornwell 1989), and the polarization degree is higher on both sides (Perlman et al. 1999). It is suggested again that the field involved may be a helical magnetic field.

The characteristic of the FRM distribution we see in Figure 1 and in a number of other cases is that the value of the FRM has a systematic gradient across the small width of the jets. In other words, the FRM has opposite signs on both sides of the jet if we subtract the smoothly varying, probably foreground, contributions. It is not likely that the large-scale, foreground magnetic field distribution has a very thin, sheared layer, coincidentally perfectly parallel to our line-of-sight to the length of the projected jet. Therefore, the features in the distribution of FRM in the jets and tails having the scale of the width of the jet or tail are most likely to be due to the outer skin of the jet itself, and it is suggested that the magnetic field in the skin of the jet has systematically opposite polarity in the line-of-sight component on both sides of the jet and tail. 

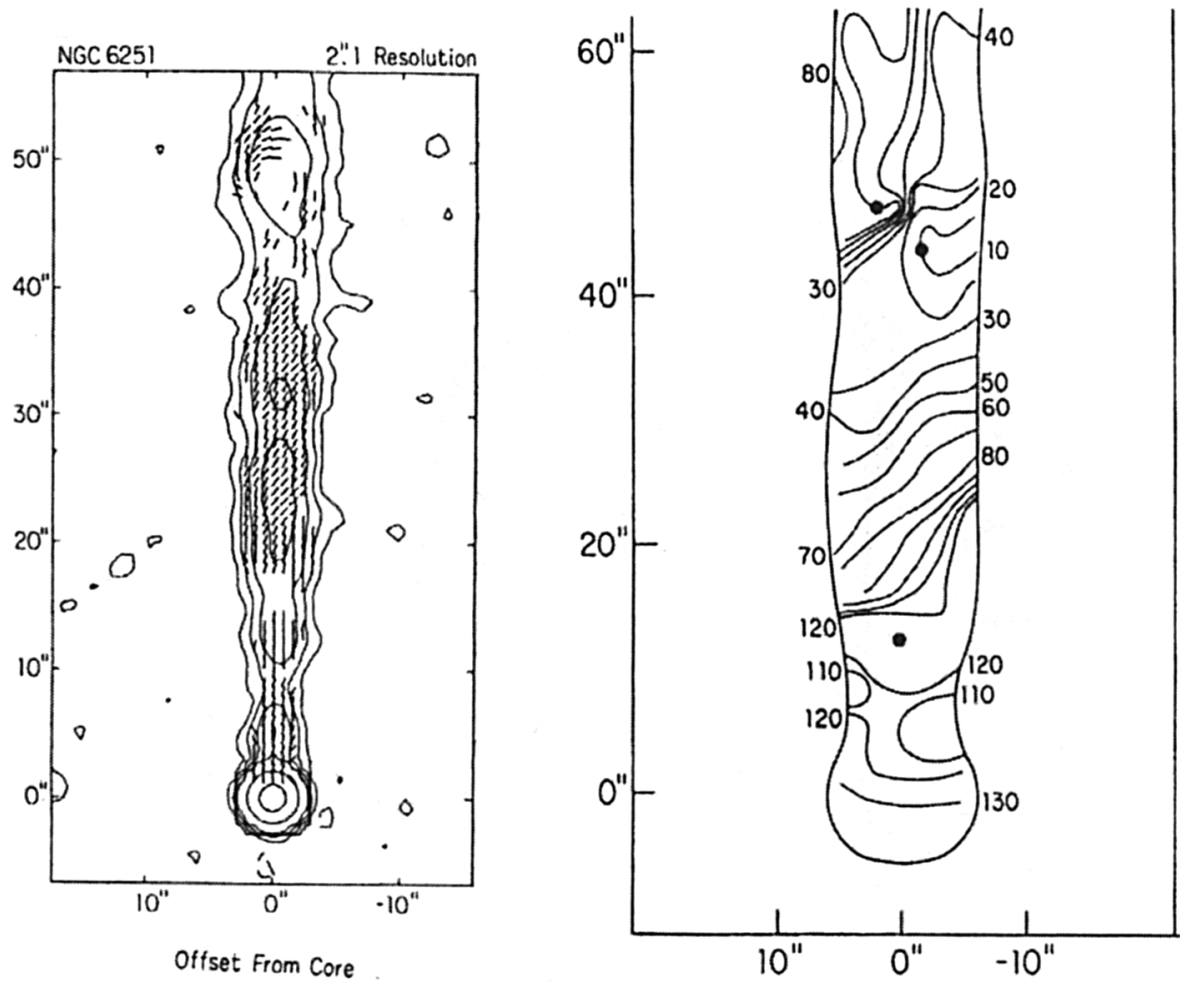

Figure 1. The distributions of (a) the projected magnetic field and (b) the FRM in NGC6251 (Perley et al. 1984). The projected field vectors are tilted from the jet axis, and the FRM distribution is asymmetric with respect to the values along the axis, suggesting that the line-of-sight magnetic field has opposite signs on both sides. The magnetic field involved is likely to have a helical configuration.

This, combined with the fact that the projected directions of the magnetic field inside the jet are systematically tilted, suggests that what we are seeing may be a helical magnetic field in the sheath of the jet.

\subsection{Structural Helix Due to Helical Kink Instability?}

We have proposed measuring the distribution of FRM and the direction of the FRM-corrected projected magnetic field very close to the AGN core to derive the information about the 3D structure of the magnetic field in the jets near the AGN cores, corresponding to those features mentioned above, by using Halca and the VLBA. It has been delayed due to the instrumental trouble of Halca.

However, some of the corresponding data for larger-scale structures have already been obtained, and we can use them to get some insight about the $3 \mathrm{D}$ structure of the magnetic field of the large-scale jets and tails. For example, 


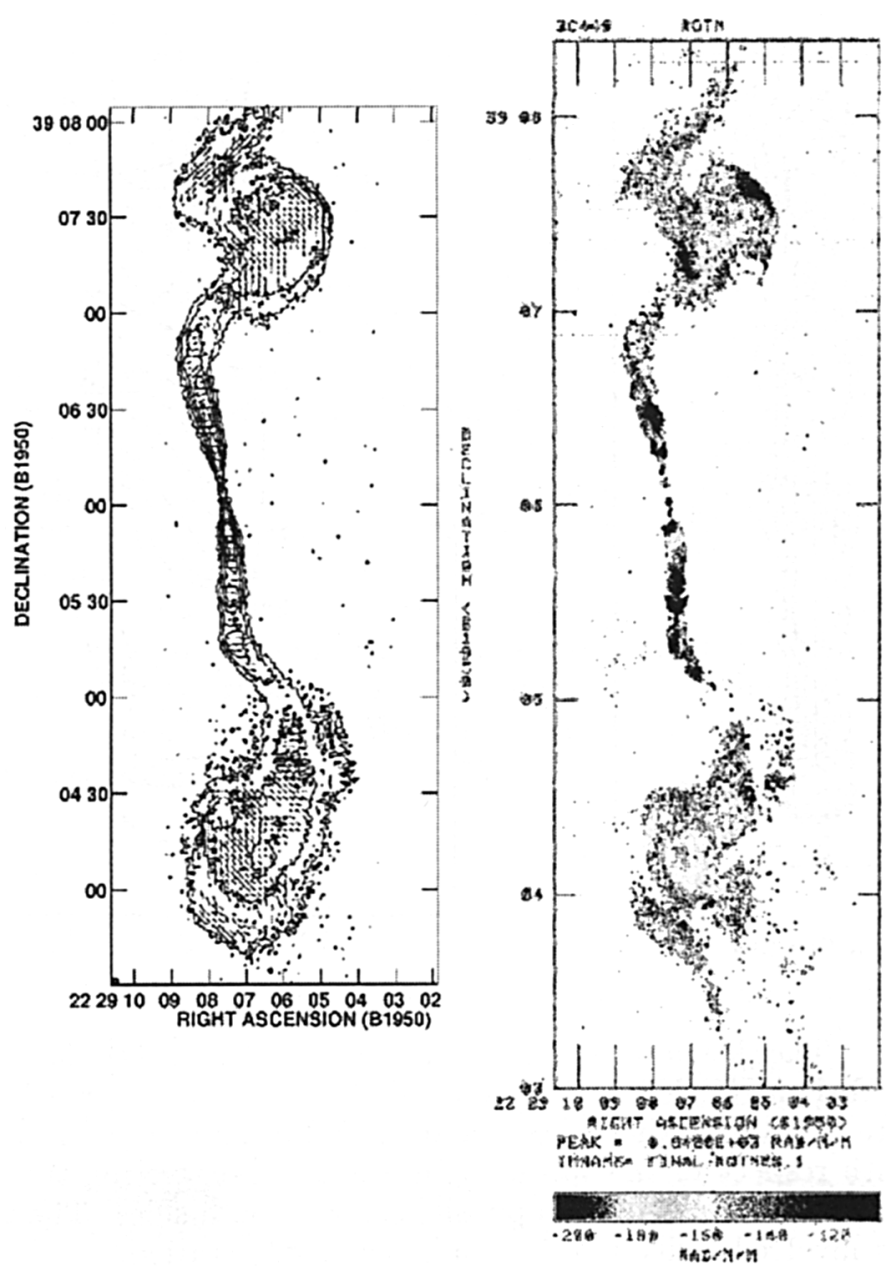

Figure 2. (a) The projected magnetic field vector and (b) FRM of 3C449 (Feretti et al. 1999). It is seen that the FRM shows a very regular distribution that is correlated with the wiggle. We can attribute these to a structural helix corresponding to the magnetic helical kink.

Feretti et al. (1999) obtained a very systematic distribution of the FRM along the wiggled structure of the AGN jets in 3C449, together with the FRM-corrected distribution of the directions of magnetic field, as shown in Figures 2a, b. The regularity in the distribution of FRM in a good correlation with the wiggling is very clear, and it is not possible that this pattern is due to the foreground medium independent of the jet.

Indeed, it is true that the FRM is basically due to the "foreground" medium in between the strongly emitting source and the observer, integrated along the 
line-of-sight all the way (cf. Eilek \& Owen 2000). In some cases, however, the contribution from the skin of the jet structure itself can be the strongest contribution to the FRM. The skin of the jet itself can play the role of the Faraday rotator to the radiation coming from the inner part of the jet in which the greater flux of the high-energy electrons is preferentially injected from the engine, since the skin of the jet itself can be the only part having a systematic field with a structure as fine as the jet width and correlated to the features of the jet like its bending. The surrounding, large-scale fields will result in a FRM with a smoother, large-scale pattern covering the entire width of the narrow jets.

Another example of the beautiful distribution of the FRM and the FRMcorrected directions of the projected magnetic field is that of 3C465 (Eilek \& Owen 2000). The FRM-corrected directions of the projected magnetic field are very nicely correlated with the structural wiggle, like the straw in the big, bent rope of straw in front of a Shinto shrine, and the FRM distribution in the jet and tail has a winding structure having a width of that of the tail itself.

Since it is not likely that such systematic structures in the jets and tails can be created by the actions of some unknown mechanism in each locality (which do not have a phase correlation), we conclude that such a systematic structure should be due to an effect coming from the powerful AGN core itself. From the shape of the distribution, we may guess that the effect comes in the form of magnetic twists, or large-amplitude, torsional Alfvén wave trains (TAWTs), propagating out along the large-scale magnetic field. In other words, the characteristic structure of the magnetic field in the outer parts of the jets and tails is likely to be due to winding up by the powerful central engine - the accretion disk rotating around the central massive black hole, in our picture.

Such a magnetic twist, or large-amplitude, torsional Alfvén wave train, may also be able to explain (1) the large energy of hotspots and lobes as coming from the central engine in the form of the Poynting flux plus the flux of accelerated high-energy particles along the strengthened, large-scale magnetic field connected to the central engine, and, (2) the helical structure in the shape of the jets as due to helical kink instability where the toroidal component exceeds the Krascal-Shafranov limit. (3) The large-scale (intergalactic-scale) magnetic field near the jets, which was not measured directly probably because it is weak, will be very naturally explained as having been pinched into the jets by the "sweeping pinch" effect of the TAWT, all as suggested by the magnetodynamic model of Uchida \& Shibata (1985). The application to AGN cases is described elsewhere in these Proceedings but summarized very briefly in the following.

\section{Models for Jets Proposed Thus-far}

Now, we very briefly discuss models thus-far advocated. It may be said that the nonmagnetic models dealing with the purely gas-dynamical jets seem to fail to explain many of the observations suggesting the intrinsic relevance of the magnetic field. As a clear example, the systematic magnetic structure will not be explained by the nonmagnetic, gas-dynamic models.

Next, there is a category of magnetic models in which a given rigid magnetic field is assumed to stick out at a fixed angle at a fixed location of the rotating accretion disk, exerting a centrifugal effect on the gas to be accelerated into 
a jet flow. This is an extension of the centrifugal acceleration of stellar wind, and the models by Blandford \& Payne (1982) and Pudritz \& Norman (1986) may belong to this category. Those were worthwhile in showing the importance of the centrifugal acceleration effect but may not represent the reality too well (see below), and they also did not have an explanation for why such a magnetic configuration was formed.

In this regard, the magnetodynamic model proposed by Uchida \& Shibata (1985; Shibata \& Uchida 1986) seems to be more relevant. It was originally proposed for star-formation bipolar jets (molecular outflows and optical jets) and extended to AGN radio jets (Uchida \& Shibata 1986; Matsumoto et al. 1997; Uchida et al. 1999; Nakamura et al., in preparation). In this model, we consider that a part of the primordial gas, having a weak, large-scale magnetic field and some angular momentum, contracts gravitationally towards the center and forms a central condensation and, eventually, the central gravitator plus accretion disk at the center of it.

The following processes occur in the central part of our model. (1) The magnetic field is brought into the central condensation and strengthened to an appreciable value (but still passive) in the disk, where it will exert magnetic braking on the rotation of the disk, enhance accretion, and, therefore, enhance gravitational energy liberation at the central black hole. (2) The magnetic field lines continuously pulled into the azimuthal direction by the disk rotation in this process will wind up the large-scale magnetic field outside the disk and create magnetic twists (large-amplitude, torsional Alfvén wave trains $=$ TAWTs) that propagate in two directions along the large-scale magnetic field. (3) This magnetic twist, as it propagates, pinches the large-scale field which has been made into an hourglass shape in the contraction of the mass to the central region and creates thin, pinched structures extending in the bipolar directionsnamely, the "jets" with tails and the lobes with hotspots. It is shown that the "sweeping pinch" drives the gas of the disk corona into spinning gas jets and also re-accelerates the seed high-energy particles produced in the magnetic reconnection process occurring at the innermost part of the accretion disk into much higher energies by the surf-riding process. (4) The action of the "sweeping pinch" will make the lobes by tucking up the initially hourglass-shaped field and impinging onto the border of the "cavity" from which the mass contracted to form the central condensation. It will produce a strong pinching and helical instability due to the accumulation of the toroidal field. These explain the hotspots and the wiggled structures of the tail, respectively.

\section{Discussion}

The energy in our process is carried along the "jet" structure mainly by the Poynting flux of the TAWT and the flow of high-energy particles that are reaccelerated in interacting with the TAWT. A spinning gas jet is produced by the TAWT near the central engine, driven in the sweeping pinch. The main energy carrier, the TAWT, propagates near the light velocity from a certain distance outwards, since it tends to a circularly polarized, "large-amplitude electromagnetic wave" when it propagates in the low-density region. It may correspond to the torsional-mode version of the "extremely low-frequency, magnetic dipole emission" of large-amplitude electromagnetic waves emitted by a pulsar beyond 


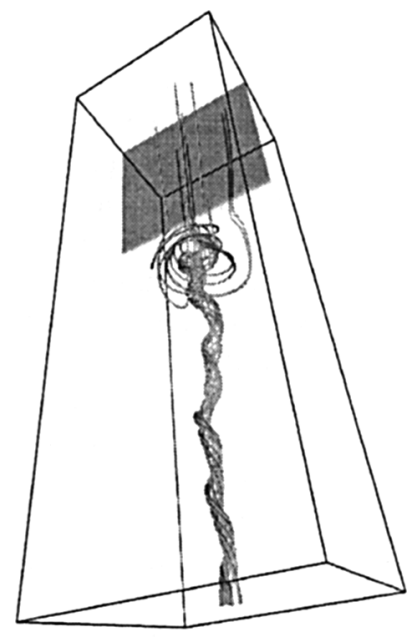

Figure 3. Development of the helical instability when the TAWT in our model encounters the border of the "cavity", and the toroidal field accumulates.

the light-cylinder. In our model, the seed high-energy electrons produced in the magnetic reconnection at the colliding point of the "avalanches" at the innermost part of the accretion torus will be re-accelerated to higher energies by surf-riding on the successively emitted TAWTs in the above-mentioned process.

When the TAWT approaches the border of the "cavity" from which the primordial gas contracted toward the central body, a strong accumulation of the toroidal component of the field will occur due to the decrease in the Alfvén velocity. Especially strong pinching will be caused when the tip of the jet impinges on the low-Alfvén-velocity region. The observed "hotspot" is caused by the electrons re-accelerated by the Fermi I process between the relatively approaching magnetic mirrors (TAWTs) and eventually dumped into this strongly pinched region, and very strong synchrotron emission will be emitted there.

This accumulation of the toroidal field and the redistribution of it on bouncing from the hotspot region may cause a helical instability of the jet structure near the end points. We attribute the wiggled structure of the observed radio jets to this structural helix caused by helical kink instability.

We have been describing a nonrelativistic case. A relativistic treatment is desirable and has been performed by Koide, Shibata, \& Kudoh (1998), and some results have been reported elsewhere in these Proceedings (Shibata, Koide, \& Kudoh 2000). The Lorentz factor of the gas flow in the jets, however, will not be as high as recent observations require, because the velocity of the gas flow is restricted by the Keplerian velocity of the innermost part of the disk (Kudoh \& Shibata 1997) at the last stable orbit at $3 R_{S c h}$. The rotation nearer the black hole may be faster but heavily Doppler shifted towards slower rotation when seen from outside. 
The basic property of our model described above is the action of a TAWT, a large-amplitude electromagnetic wave in the vacuum limit, and the gas jet may be regarded as a byproduct of the sweeping pinch and the centrifugal effect. The main energy carrier, the TAWT carrying energy in the form of Poynting flux, will tend to a large-amplitude, circularly polarized electromagnetic wave in the low-density region and propagates near the speed of light. This is good for the problem of the propagation distance during the lifetime of the accretion disk, and this large-amplitude electromagnetic wave may be able to explain the electron re-acceleration to extremely high energy, just as in the case of a pulsar's low-frequency, large-amplitude electromagnetic wave due to magnetic dipole emission. It may not be necessary to accelerate the gas jet as a whole to an energy with the Lorentz-factor as large as $10^{3}$. The superrelativistic particles explaining observed $\gamma$-rays may be either due to the re-accelerated particles in the process mentioned above or due to other process that may be caused by some more intricate magnetoplasma-dynamic effects, such as a plasma physical "double layer" that corresponds to the "gap" in the pulsar physics. In the much better-known, solar high-energy phenomena, the high-energy particles emitting $\gamma$-rays are accelerated by the action of magnetic field in the low- but greaterenergy magnetodynamic phenomena, and not the reverse.

As for the measurements giving weak magnetic field values, it is also wellknown that the magnetic field values derived can be very much smaller than the true values if measured with insufficient spatial resolution. There also exists a tendency for the high-energy particles to avoid strong field regions.

Remarkable evidence for the presence of very strong, systematic magnetic field near the AGN core may be the bent path of a superluminal source (an example is the superluminal source in 3C120: Walker, Benson, \& Unwin 1987) which is initially ejected at a certain direction away from the axis of the largescale jet, but, eventually, its path is bent to the direction of the large-scale jet. This is not thinkable without a strong magnetic field.

Finally, we consider the accretion disk as the central engine and not the spinning black hole itself. The TAWT will not be able to come out of the black hole, since it is a direct signal of the rotation of the black hole and should not be able to cross the event horizon, just as photons can not. Types of mechanisms like Blandford-Znajek's would actually require a driver of the process. A more likely process occurring may be that the mass rotating at the innermost region of the disk sends a TAWT outwards along the external field, and a paired TAWT will propagate down into the black hole with the infalling mass.

\section{References}

Blandford, R. D., \& Payne, D. G. 1982, MNRAS, 119, 883

Eilek, J., \& Owen, F. 2000, ApJ, submitted

Feretti, L., Perley, R., Giovanni, G., \& Andernach, H. 1999, A\&A, 341, 29

Koide, S., Shibata, K., \& Kudoh, T. 1998, ApJ, 495, L63

Kudoh, T., \& Shibata, K. 1997, ApJ, 474, 362

Matsumoto, R., Uchida, Y., Hirose, S., Shibata, K., Hayashi, M., Ferrari, A., Bodo, G., \& Norman, C. 1997, ApJ, 461, 115

Nakamura, M., Uchida, Y., \& Hirose, S., in preparation 
Owen, F., Hardee, P., \& Cornwell, T. 1989, ApJ, 340, 698

Perley, R., Bridle, A., \& Willis, A. 1984, ApJS, 54, 291

Perlman, E., Biretta, J., Zhou, F., Sparks, W., \& Macchetto, F. 1999, AJ, 117, 2185

Pudritz, R. E., \& Norman, C. A. 1986, ApJ, 301, 571

Shibata, K., Koide, S., \& Kudoh, T. 2000, these Proceedings

Shibata, K., \& Uchida, Y. 1986, PASJ, 38, 631

Uchida, Y., Nakamura, M., Hirose, S., \& Uemura, S. 1999, A\&AS, 264, 195

Uchida, Y., Nakamura, M., Miyagoshi, T., Kobayashi, T., Mukawa, T., \& Hirose, S. 2000, these Proceedings

Uchida, Y., \& Shibata, K. 1985, PASJ, 37, 515

Uchida, Y., \& Shibata, K. 1986, Can. J. Phys., 64, 507

Walker, R., Benson, J., \& Unwin, S. 1987, ApJ, 316, 546 